\title{
PREPARAÇÃO DE $\alpha$-ACETILOXI-N-CICLOEXILAMIDAS ATRAVÉS DA REAÇÃO DE PASSERINI UTILIZANDO DIMETILCARBONATO COMO SOLVENTE AMBIENTALMENTE AMIGÁVEL
}

\author{
Bárbara R. Oliveira, ${ }^{a}$ Carla C. Silva, ${ }^{a}$ Juliana C. P. Calado, ${ }^{a}$ Wagner L. Batista, ${ }^{a}$ Fernanda A. Siqueira ${ }^{\mathrm{b}}$ e Luiz S. Longo Jr. ${ }^{\mathrm{a}, *}$ \\ aDepartamento de Ciências Farmacêuticas, Instituto de Ciências Ambientais, Químicas e Farmacêuticas, Universidade Federal de \\ São Paulo, Campus Diadema, Rua São Nicolau, 210, 09913-030 Diadema - SP, Brasil \\ bDepartamento de Química, Instituto de Ciências Ambientais, Químicas e Farmacêuticas, Universidade Federal de São Paulo, \\ Campus Diadema, Rua São Nicolau, 210, 09913-030 Diadema - SP, Brasil
}

Recebido em 31/03/2017; aceito em 15/08/2017; publicado na web em 27/10/2017

\begin{abstract}
PREPARATION OF $\alpha$-ACETYLOXY- $N$-CYCLOHEXYLAMIDES VIA PASSERINI REACTION USING DIMETHYLCARBONATE AS ECO-FRIENDLY SOLVENT. A series of $\alpha$-acetyloxy- $N$-cyclohexylamides was efficiently obtained via Passerini reaction of cyclohexyl isocyanide, acetic acid, and different aromatic and aliphatic aldehydes using refluxing dichloromethane or dimethylcarbonate (DMC). Reactions performed with DMC led to desired products in moderate to good yields, corroborating this solvent as a eco-friendly alternative to the chlorinated solvents commonly used in this transformation. The $\alpha$-acetyloxy- $N$-cyclohexylamides thus obtained were tested against Escherichia coli, Bacillus cereus, Pseudomonas aeruginosa, and Staphylococcus aureus, as well as promastigote forms of $L$.(L.) amazonensis. The $\alpha$-(Acetyloxy)- $N$-cyclohexyl-pentanamide showed low antibacterial activity against Gram-positive ( $S$. aureus and B. cereus) and Gram negative (E. coli and P. aeruginosa) bacteria (MIC $=8,3-16,5 \mathrm{mmol} \mathrm{L}^{-1}$ ), while $\alpha$-(acetyloxy)- $N$-cyclohexyl-4-bromo-benzeneacetamide showed leishmanicidal activitity $\left(\mathrm{IC}_{50}=0,099 \mathrm{mmol} \mathrm{L}^{-1}\right)$.
\end{abstract}

Keywords: acetyloxy- $N$-cyclohexylamides; Passerini reaction; dimethylcarbonate; green chemistry; antibacterial activity; leishmanicidal activity.

\section{INTRODUÇÃO}

Reações multicomponentes (multicomponent reactions, MCRs) são aquelas onde três ou mais materiais de partida reagem em uma única operação, num mesmo frasco reacional, sem isolamento de intermediários reacionais, para dar um produto onde a maioria dos átomos dos reagentes (senão todos) está incorporada na estrutura molecular do produto final. ${ }^{1}$ Tais reações são, portanto, processos com elevada eficiência atômica. A primeira reação multicomponente foi descrita em 1850 por A. Strecker ${ }^{2}$ para a síntese de $\alpha$-aminonitrilas a partir de um composto carbonílico, uma amina e o íon cianeto. Esta reação tornou-se importante uma vez que as $\alpha$-aminonitrilas obtidas podem ser facilmente hidrolisadas aos $\alpha$-aminoácidos correspondentes. Desde então, várias outras reações multicomponentes clássicas foram desenvolvidas, com destaque para a reação de Biginelli na síntese de 3,4-diidropirimidin-2(1H)-onas, ${ }^{3}$ a reação de Hantzsch para a síntese de 1,4-diidropiridinas ${ }^{4}$ e a reação de Mannich para a síntese de compostos $\beta$-aminocarbonílicos, ${ }^{5}$ dentre muitas outras (Figura 1). Muitas reações multicomponentes são de grande interesse para a pesquisa e desenvolvimento de novos fármacos, uma vez que são capazes de gerar grande diversidade molecular e complexidade estrutural em uma única etapa. ${ }^{6}$ Como são transformações químicas de relativa simplicidade operacional, as MCRs podem ser facilmente automatizadas (Síntese Paralela), tornando-as ferramentas úteis para a Química Combinatória na obtenção de bibliotecas de moléculas orgânicas de baixo peso molecular com potencial atividade biológica, a qual pode ser avaliada para um grande número de entidades moleculares via HTS (high throughput screening). ${ }^{7}$

Vários artigos de revisão sobre MCRs podem ser encontrados na literatura, cobrindo diferentes aspectos e tipos destas reações. ${ }^{8}$ Podemos destacar a revisão recente de Cioc et al., ${ }^{9}$ os quais discutem

\footnotetext{
*e-mail: luiz.longo@unifesp.br
}

as MCRs como ferramentas úteis para síntese orgânica mais sustentável, e revisão de $\mathrm{Y} \mathrm{Gu}^{10}$ sobre as várias estratégias desenvolvidas para MCRs em meios não convencionais. As reações multicomponentes também se mostraram muito úteis na síntese de heterociclos de baixo peso molecular, estruturas privilegiadas e presentes em moléculas de vários compostos orgânicos com propriedades biológicas relevantes, sejam eles produtos naturais ou moléculas sintéticas candidatas a fármacos. ${ }^{11}$

As reações multicomponentes que utilizam isonitrilas (isocyanide-based multicomponent reactions, IMCRs) como um dos materiais de partida são particularmente interessantes, devido à disponibilidade comercial de várias isonitrilas e à grande variedade de transformações possíveis de serem realizadas com estes compostos. ${ }^{12} \mathrm{O}$ grupo $-\mathrm{NC}$ apresenta uma reatividade química muito peculiar, conferindo à molécula um sítio nucleofílico e outro eletrofílico. Por esse motivo, as isonitrilas estão presentes em um grande número de reações multicomponentes conhecidas até hoje e surgidas após os trabalhos pioneiros de Passerini ${ }^{13}$ e Ugi $^{14}$ (Figura 1).

A reação de Passerini foi inicialmente descrita em $1920 .{ }^{13}$ Nesta transformação, um composto carbonílico (geralmente um aldeído) reage com um ácido carboxílico e uma isonitrila para fornecer uma $\alpha$-acilóxi-carboxamida como produto final (Figura 1). Embora conhecida há quase cem anos, as reações de Passerini ficaram esquecidas durante muito tempo, ressurgindo na década de 90 devido ao interesse nas MCRs como métodos eficientes para construção de quimiotecas de compostos orgânicos para screening de atividade biológica. ${ }^{15}$ Desde então, vários grupos de pesquisa têm se dedicado ao estudo das condições reacionais para esta reação orgânica. ${ }^{16}$

Considerando todos os aspectos das transformações químicas sustentáveis, tais como economia de átomos, catálise e eficiência energética, entre outros, o meio reacional tem papel muito importante. De acordo com Constable et al.,${ }^{17}$ o solvente da reação representa até $80 \%$ do total de toda a massa de materiais para os processos 

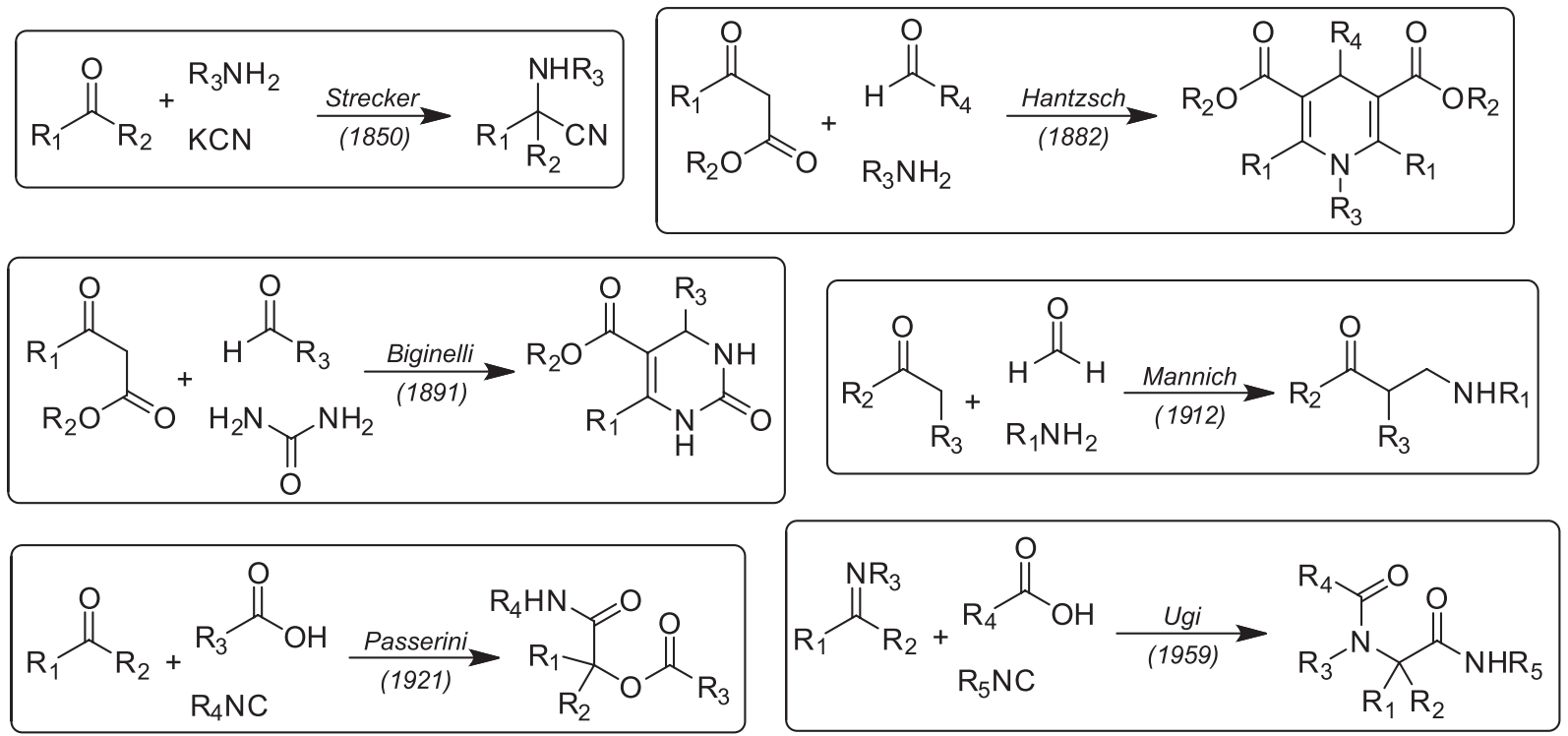

Figura 1. Principais Reações Multicomponentes

químicos de produção de Ingredientes Farmacêuticos Ativos (Active Pharmaceutical Ingredient, API). Diante disso, grandes indústrias farmacêuticas como a Pfizer, ${ }^{18}$ a GSK, ${ }^{19}$ e a Sanofi ${ }^{20}$ desenvolveram recentemente guias de seleção de solventes para processos químicos aplicados à síntese de APIs.

Os solventes reacionais são responsáveis por modificações na reatividade de reagentes, catalisadores e produtos, pelos fenômenos de transferência de massa e de calor, assim como pela estabilização de estados de transição. Além disso, os solventes orgânicos são frequentemente utilizados em processos de extração, lavagem e purificação dos produtos das reações, assim como nos processos de purificação por recristalização ou cromatografia, sendo utilizado também para efetuar limpeza de equipamentos. Portanto, o solvente tem um impacto enorme em métricas verdes das reações orgânicas industriais.

Os carbonatos orgânicos constituem solventes dipolares apróticos, assim como DMSO e DMF, que vêm ganhando destaque recente como solventes úteis para reações orgânicas. ${ }^{21,22}$ Carbonatos acíclicos como o dimetilcarbonato (DMC) e o dietilcarbonato (DEC) apresentam propriedades semelhantes às de alguns solventes convencionais, tais como o diclorometano e THF. Sua disponibilidade em grande quantidade a custo relativamente baixo, juntamente com o fato de serem biodegradáveis e de baixa toxicidade, entre outras, são características que permitem seu uso como alternativas aos solventes orgânicos voláteis tradicionais. Neste trabalho, descrevemos o uso do dimetilcarbonato como solvente alternativo em reações de Passerini, sob aquecimento convencional e por irradiação de micro-ondas, na preparação de uma série de $\alpha$-acetiloxi- $N$-cicloexilamidas com potencial atividade antibacteriana e leishmanicida.

\section{RESULTADOS E DISCUSSÃO}

Os carbonatos orgânicos comumente utilizados como solventes em reações orgânicas, e com boa disponibilidade comercial a partir de vários fornecedores, são o dimetilcarbonato (DMC), dietilcarbonato (DEC), etileno carbonato (EC) e propileno carbonato (PC) (Figura 2). ${ }^{23}$<smiles>[R]OC(=O)O[R]</smiles>

Figura 2. Carbonatos orgânicos disponíveis comercialmente

A Tabela 1 sumariza algumas propriedades físico-químicas e toxicológicas dos principais carbonatos orgânicos. ${ }^{21}$ Os carbonatos cíclicos - EC e PC - são menos empregados como solventes orgânicos devido ao fato de possuírem elevado ponto de ebulição $\left(247,9{ }^{\circ} \mathrm{C}\right.$ e $245,9^{\circ} \mathrm{C}$, respectivamente) e alta viscosidade, o que certamente impõe dificuldades para sua remoção por destilação a pressão reduzida na etapa de isolamento dos produtos de reação. Já o DMC e DEC são mais comumente utilizados como solventes em várias transformações orgânicas, ${ }^{21}$ apresentam pontos de ebulição menores que os carbonatos cíclicos, baixa solubilidade em água e elevada constante dielétrica, sendo alternativas a solventes orgânicos dipolares apróticos como DMSO e DMF. O ponto de ebulição do DMC é de cerca de

Tabela 1. Propriedades físico-químicas e toxicológicas dos carbonatos orgânicos ${ }^{a}$

\begin{tabular}{|c|c|c|c|c|}
\hline & $D M C$ & $D E C$ & $E C$ & $P C$ \\
\hline Ponto de ebulição $\left({ }^{\circ} \mathrm{C}\right)$ & $89,9^{a}$ & $125,9^{a}$ & $247,9^{b}$ & $245,9^{b}$ \\
\hline Densidade a $20^{\circ} \mathrm{C}\left(\mathrm{g} / \mathrm{cm}^{3}\right)$ & $1,07^{a}$ & $0,98^{a}$ & $1,34^{b}$ & $1,20^{b}$ \\
\hline Viscosidade a $25^{\circ} \mathrm{C}(\mathrm{cP})$ & $0,590^{a}$ & $0,753^{c}$ & $2,56^{b}$ & $2,50^{b}$ \\
\hline Biodegradabilidade $\left(\mathrm{d}^{-1}\right)^{b}$ & $88 \%$ & $75 \%$ & $100 \%$ & $94 \%$ \\
\hline $\mathrm{LC}_{50}$ (inalação, rato) $\left(\mathrm{mg} \mathrm{L}^{-1}\right)^{e}$ & $>140(4 \mathrm{~h})$ & nd & nd & nd \\
\hline $\mathrm{LC}_{50}$ (oral, rato) $\left(\mathrm{mg} \mathrm{kg}^{-1}\right)^{e}$ & 9000 & $>4900$ & $>5000$ & 34920 \\
\hline $\mathrm{LC}_{50}($ peixe $)\left(\mathrm{mg} \mathrm{L}^{-1}\right)^{c}$ & 1000 & nd & $>1000$ & 2200 \\
\hline
\end{tabular}

(a) Dados obtidos da referência 21; (b) Protocolos OECD 301E e 302B; (c) Teste realizado com Leuciscus idus (96 h); nd = não disponível. 
$90{ }^{\circ} \mathrm{C}$, e este tem uma excelente biodegradabilidade se comparada ao diclorometano, fazendo deste carbonato um solvente ambientalmente amigável ideal para substituição de solventes clorados. ${ }^{24}$

O DMC é um solvente com razoável estabilidade térmica e química, podendo ser armazenado em condições ambientes, sem necessidade de atmosfera inerte, e com boa resistência a hidrólise. Se necessário seu emprego em condições anidras, entretanto, tratamento de secagem por métodos usuais são necessários, a exemplo de outros solventes orgânicos ordinários. O perfil toxicológico do DMC, comparado ao diclorometano, um solvente clorado comum em reações orgânicas, é bastante superior. Por exemplo, a $\mathrm{LD}_{50}$ por inalação em ratos é maior que $140 \mathrm{mg} \mathrm{mL}^{-1}$ (4h) para o DMC, enquanto para o $\mathrm{CH}_{2} \mathrm{Cl}_{2}$ é de cerca de $88 \mathrm{mg} \mathrm{mL}^{-1}\left(0,5\right.$ h). ${ }^{21} \mathrm{Já}$ a $\mathrm{LD}_{50}$ oral em ratos para o DMC é de $9000 \mathrm{mg} \mathrm{kg}^{-1}$ e para o $\mathrm{CH}_{2} \mathrm{Cl}_{2}$ é de $1600 \mathrm{mg} \mathrm{kg}^{-1}$. $^{21}$

Entendendo a importância do papel do solvente em reações orgânicas, e sabendo que o diclorometano é um solvente bastante empregado em reações multicomponentes, decidimos testar a utilidade do dimetilcarbonato como um possível substituto para este solvente clorado na reação de Passerini para a preparação de $\alpha$-acetiloxi- $N$-cicloexilamidas. Para isto, uma reação modelo foi estudada com uma mistura equimolar de cicloexilisonitrila, benzaldeído e ácido acético, levando ao produto desejado 1 em apenas $55 \%$ e $28 \%$ de rendimento após $48 \mathrm{~h}$ de refluxo em $\mathrm{CH}_{2} \mathrm{Cl}_{2}$ ou DMC, respectivamente (Esquema 1). É importante chamar atenção para o fato de que tempos reacionais menores levaram a resultados erráticos, com consumo parcial dos materiais de partida e rendimentos não reprodutíveis. Ainda é importante ressaltar a diferença de temperatura em que as reações foram realizadas, dada a diferença entre os pontos de ebulição dos dois solventes em questão.<smiles>N#CC1CCCCC1</smiles>

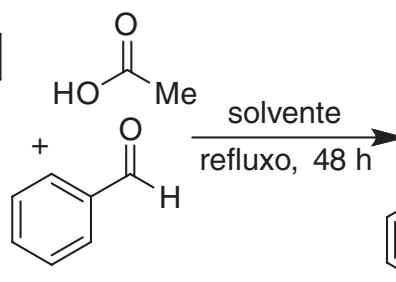<smiles>CC(=O)OC(C(=O)NC1CCCCC1)c1ccccc1</smiles><smiles>[AlH2]</smiles>

$$
\begin{aligned}
& \mathrm{CH}_{2} \mathrm{Cl}_{2}(55 \%) \\
& \operatorname{DMC}(28 \%)
\end{aligned}
$$

Esquema 1. Reação de Passerini modelo efetuada em $\mathrm{CH}_{2} \mathrm{Cl}_{2}$ e DMC como solventes

Em seguida, variamos o componente aldeído da reação de Passerini, utilizando benzaldeídos para-substituídos com diferentes grupos doadores e retiradores de elétrons, assim como aldeídos alifáticos (Tabela 2). Como esperado, aldeídos mais reativos, tais como o para-nitrobenzaldeído e os butiraldeídos, levaram às $\alpha$-acetiloxi- $N$-cicloexilamidas desejadas em rendimentos maiores, em ambos os solventes. Por exemplo, a reação do para-nitrobenzaldeído nas condições estudadas levou a 3 em $86 \%$ e $80 \%$ de rendimento quando a reação foi efetuada em $\mathrm{CH}_{2} \mathrm{Cl}_{2} \mathrm{e} \mathrm{DMC}$, respectivamente (entrada 3). Já quando o para-anisaldeído foi utilizado como material de partida, o composto 2 foi obtido em rendimentos baixos, $36 \%$ para $\mathrm{CH}_{2} \mathrm{Cl}_{2} \mathrm{e}$ $25 \%$ para DMC (entrada 2).

Encorajados pelos rendimentos comparáveis das reações efetuadas em refluxo de $\mathrm{CH}_{2} \mathrm{Cl}_{2} \mathrm{e}$ DMC, estendemos o estudo para uma série de benzaldeídos orto-substituídos, os quais foram refluxados com cicloexilisonitrila e ácido acético em DMC por 48 horas, fornecendo as respectivas $\alpha$-acetiloxi- $N$-cicloexilamidas 7-10 em rendimentos que variaram de bons a excelentes (Esquema 2).
Tabela 2. Reação de Passerini para obtenção das $\alpha$-acetiloxi- $N$-cicloexilamidas (1-6) ${ }^{\mathrm{a}}$

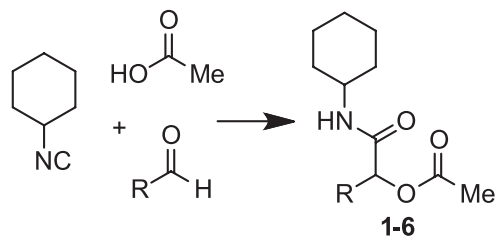

\begin{tabular}{lccc}
\hline Entrada & $\mathrm{R}$ (composto) & Rend. (A) & Rend. (B) \\
\hline 1 & $\mathrm{C}_{6} \mathrm{H}_{5}(\mathbf{1})$ & $55 \%$ & $28 \%$ \\
2 & $4-\mathrm{OMe}^{\mathrm{b}} \mathrm{C}_{6} \mathrm{H}_{4}(\mathbf{2})$ & $36 \%$ & $25 \%$ \\
3 & $4-\mathrm{NO}_{2}-\mathrm{C}_{6} \mathrm{H}_{4}(\mathbf{3})$ & $86 \%$ & $80 \%$ \\
4 & $4-\mathrm{Br}^{\mathrm{b}} \mathrm{C}_{6} \mathrm{H}_{4}(\mathbf{4})$ & $55 \%$ & $49 \%$ \\
5 & $n-\mathrm{C}_{3} \mathrm{H}_{7}(\mathbf{5})$ & $75 \%$ & $66 \%$ \\
6 & $i-\mathrm{C}_{3} \mathrm{H}_{7}(\mathbf{6})$ & $85 \%$ & $81 \%$ \\
\hline
\end{tabular}

(a) Reagentes e Condições: CONDIÇÃO A: cicloexilisonitrila (1,0 mmol), ácido acético $(1,0 \mathrm{mmol})$ e aldeído $(1,0 \mathrm{mmol}), \mathrm{CH}_{2} \mathrm{Cl}_{2}(5 \mathrm{~mL})$, refluxo, 48 horas; CONDIÇÃO B: cicloexilisonitrila $(1,0 \mathrm{mmol})$, ácido acético $(1,0$ $\mathrm{mmol})$ e aldeído (1,0 mmol), DMC $(5 \mathrm{~mL})$, refluxo, 48 horas; $b$ Produto 2 obtido em mistura com para-anisaldeído de partida, na proporção de 4:1, respectivamente (determinado por $\mathrm{RMN}^{1} \mathrm{H}$ ); (b) Rendimentos isolados.

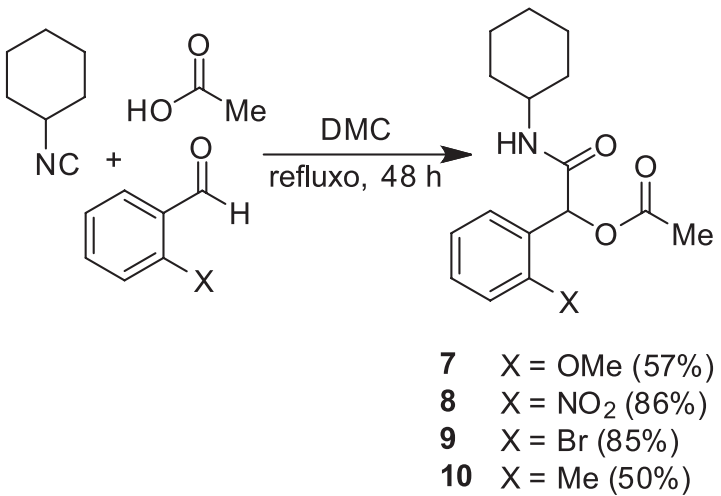

Esquema 2. Reação de Passerini com a série de orto-benzaldeídos realizada em refluxo de DMC

Também investigamos o uso da radiação de micro-ondas como fonte alternativa de aquecimento para as reações de Passerini estudadas. Em 2011, Barreto e colaboradores prepararam uma série de $\alpha$-acilóxi carboxamidas através da Reação de Passerini sem solvente aquecida por irradiação de micro-ondas. ${ }^{25}$ Mais recetemente, Ingold e colaboradores descreveram a síntese de análogos furoxânicos do tocferol empregando reações de Passerini em condições sustentáveis. ${ }^{26}$

Tomamos como modelo a reação entre o orto-nitrobenzaldeído, cicloexilisonitrila e ácido acético em DMC, utilizando diferentes temperaturas e tempos reacionais, em tubo aberto (com dispositivo de refluxo do solvente, quando necessário) e em tubo fechado. Os resultados estão sumarizados na Tabela 3. Quando as reações foram feitas em tudo aberto sob refluxo de DMC por 1 hora (reator Discover®, CEM), a $\alpha$-acetiloxi- $N$-cicloexilamida 8 foi obtida em rendimentos ligeriamente inferiores àqueles obtidos em refluxo de DMC sob aquecimento convencional (entradas 1-4). É importante ressaltar que nos casos onde não se observou conversão completa dos materiais de partida, também não houve isolamento de subprodutos reacionais, e que tempos de reação maiores que os especificados na Tabela 3 não resultaram em incremento do rendimento da reação. Entretanto, quando a mesma reação foi efetuada em sistema fechado (Microwave $300 \circledR$, Anton Paar), o que permitiu trabalhar em temperaturas acima 
do ponto de ebulição do DMC, o composto 8 foi isolado em $82 \%$ e $85 \%$ de rendimento após 1 hora de reação a $130{ }^{\circ} \mathrm{C}$ (entradas 6$)$ e a $150{ }^{\circ} \mathrm{C}$ (entrada 7), respectivamente.

Tabela 3. Estudo modelo para as reações de Passerini em DMC sob aquecimento por micro-ondas. ${ }^{a}$

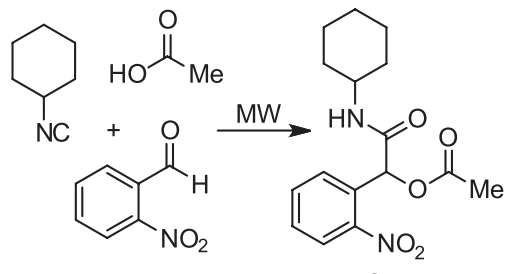

8

\begin{tabular}{|c|c|c|}
\hline Entrada & Condições & Rendimento $^{c}$ \\
\hline 1 & $\begin{array}{c}\text { Tubo aberto, DMC } \\
70^{\circ} \mathrm{C}, 1 \text { hora }\end{array}$ & $65 \%$ \\
\hline 2 & $\begin{array}{c}\text { Tubo aberto, DMC } \\
70^{\circ} \mathrm{C}, 2 \text { horas }\end{array}$ & $68 \%$ \\
\hline 3 & $\begin{array}{c}\text { Tubo aberto, DMC } \\
70^{\circ} \mathrm{C}, 3 \text { horas }\end{array}$ & $63 \%$ \\
\hline $4^{b}$ & $\begin{array}{c}\text { Tubo aberto, DMC } \\
90^{\circ} \mathrm{C}, 1 \text { hora }\end{array}$ & $72 \%$ \\
\hline 5 & $\begin{array}{c}\text { Tubo fechado, DMC } \\
110^{\circ} \mathrm{C}, 1 \text { hora }\end{array}$ & $82 \%$ \\
\hline 6 & $\begin{array}{c}\text { Tubo fechado, DMC } \\
130^{\circ} \mathrm{C}, 1 \text { hora }\end{array}$ & $82 \%$ \\
\hline 7 & $\begin{array}{c}\text { Tubo fechado, DMC } \\
150^{\circ} \mathrm{C}, 1 \text { hora }\end{array}$ & $85 \%$ \\
\hline
\end{tabular}

(a) Reagentes e Condições: cicloexilisonitrila (1,0 mmol), ácido acético (1,0 mmol) e aldeído (1,0 mmol), DMC, MW. Reações em sistema aberto foram realizadas em reator Discover ${ }^{\circledR}(\mathrm{CEM})$ e reações em sistema fechado em reator Microwave 300® (Anton Paar); (b) Foi acoplado um condensador de refluxo ao frasco de reação dentro da cavidade do reator; (c) Rendimentos isolados.

Estes testes demonstraram que as reações em micro-ondas/sistema fechado permitem obter excelentes rendimentos para a $\alpha$-acetiloxi- $N$-cicloexilamida $8 \mathrm{em}$ apenas 1 hora de reação, sendo estes comparáveis aos rendimentos obtidos quando se utilizou refluxo de DMC por $48 \mathrm{~h}$, evidenciando as vantagens já conhecidas desta forma alternativa de aquecimento térmico. ${ }^{27}$ Infelizmente, para as reações em que foram obtidos rendimentos baixos quando do emprego de refluxo de $\mathrm{CH}_{2} \mathrm{Cl}_{2}$ ou DMC por $48 \mathrm{~h}$ (Tabela 2, entradas 1 e 2), o uso do aquecimento por irradiação de micro-ondas em sistema fechado também levou a resultados insatisfatórios. Por exemplo, a reação entre cicloexilisonitrila, benzaldeído e ácido acético em tubo fechado a 150 ${ }^{\circ} \mathrm{C}$ por 1 hora levou ao produto 1 desejado em $c a .20 \%$ de conversão, determinada por cromatografia gasosa acoplada a espctrometômetro de massas (CG-EM). De modo similar, a reação entre cicloexilisonitrila, para-anisaldeído e ácido acético nas mesmas condições reacionais, levou a 2 em apenas $c a$. $4 \%$ de conversão (determinado por CG-EM).

$\mathrm{O}$ mecanismo geralmente aceito para a reação de Passerini ${ }^{28}$ está apresentado no Esquema 3. Esta é uma reação tricomponente (3-CR), onde há um ataque do carbono nucleofílico da isonitrila ao carbono eletrofílico presente no aduto I, o qual por sua vez é formado a partir da interação por ligação de hidrogênio entre os componentes ácido carboxílico e o aldeído. Posterior rearranjo no intermediário II leva ao produto da reação. É sabido que a reação de Passerini é acelerada em solventes apróticos, indicando um mecanismo predominantemente não-polar, ${ }^{29}$ embora também existam exemplos de reações de Passerini conduzidas em água como solvente. ${ }^{30}$ Recentemente, cálculos teóricos demonstraram, entretanto, que um quarto componente ácido pode estar envolvido no estado de transição, facilitando o rearranjo para fornecer o produto final, através de uma reação pseudo-tetracomponente. ${ }^{31}$<smiles>[R3]NC(=O)C([R])OC([R])=O</smiles>

Esquema 3. Mecanismo proposto para a Reação de Passerini

As $\alpha$-acetiloxi- $N$-cicloexilamidas 1-10 foram testadas em ensaios biológicos in vitro para as atividades antimicrobianas e leishmanicida. Os compostos foram testados contra as bactérias E. coli, P. aeruginosa, S. aureus e B. cereus. Os valores de CIM (Concentração Mínima Inibitória) determinados pelo teste de microdiluição estão apresentados na Tabela 4. A partir dos dados obtidos, é possível verificar que 1 e 6 apresentaram atividade contra as bactérias E. coli e B. cereus somente na concentração de $13,1 \mathrm{mmol} \mathrm{L}^{-1}$. Já $\mathbf{5}$ apresentou atividade antibacteriana contra espécies Gram-positivas (S. aureus e B. cereus) e Gram negativas (E. coli e P. aeruginosa) com valores de CIM entre 8,3-16,5 mmol L-1 . A avaliação da atividade contra $L$. (L.) amazonensis mostrou que duas $\alpha$-acetiloxi- $N$-cicloexilamidas - compostos $2 \mathrm{e}$ 4 - apresentaram atividade leishmanicida e foram capazes de inibir o crescimento (100\%) das formas promastigotas de L. (L.) amazonensis (Tabela 4). Neste ensaio, a $\alpha$-acetiloxi- $N$-cicloexilamida 4 levou ao melhor valor de $\mathrm{IC}_{50}\left(0,099 \mathrm{mmol} \mathrm{L}^{-1}\right)$, enquanto 2 também inibiu o crescimento do parasita no valor de $\mathrm{IC}_{50}$ de $0,137 \mathrm{mmol} \mathrm{L}^{-1}$.

\section{CONCLUSÃO}

Neste estudo, demonstramos a utilidade do dimetilcarbonato como solvente ambientalmente amigável para a substituição de solventes clorados comumente utilizados em reações multicomponentes do tipo Passerini. Deste modo, foi possível a obtenção de uma série de dez $\alpha$-acetiloxi- $N$-cicloexilamidas em rendimentos variáveis (de 28 a $86 \%$ ), a depender essencialmente da reatividade do aledeído de partida, utilizando condições reacionais relativamente simples como refluxo por $48 \mathrm{~h}$ na ausência de catalisadores. As reações mostraram-se mais rápidas quando realizadas em reatores de micro-ondas (tubo fechado), mas esta forma alternativa de aquecimento parece alterar apenas a duração da reação e não levar a um incremento na conversão dos materiais de partida propriamente dito. Uma triagem preliminar da atividade antibacteriana e leishmanicida dos compostos obtidos também foi realizada. Entretanto, os resultados da atividade biológica não foram evidentes, quando comparado aos controles. Interessante notar que os compostos que apresentaram atividade antimicrobiana (1, 5 e 6), apesar de baixa, foram diferentes daqueles que demonstraram potencial para atividade leishmanicida ( 2 e 4), o que indica certa seletividade de ação para os compostos testados. Além disso, apesar da atividade leishmanicida do composto 4 ser menor que o controle pentamidina (cerca de 6,6 vezes), esta ainda se encontra dentro da faixa de concentração de atividade considerada intermediária. Tal atividade poderia ser melhorada com modificações moleculares na estrutura do composto, a fim de levar ao aumento do potencial leishmanicida. Segundo Don e Ioset, ${ }^{32}$ compostos que apresentam 
Tabela 4. Atividades antimicrobiana e leishmanicida das $\alpha$-acetiloxi- $N$-cicloexilamidas 1-10

\begin{tabular}{|c|c|c|c|c|c|}
\hline \multirow{2}{*}{ Composto } & \multicolumn{4}{|c|}{$\mathrm{CIM}(\mathrm{mM})^{a}$} & \multirow{2}{*}{$\begin{array}{c}\mathrm{IC}_{50}(\mathrm{mM})^{b} \\
\text { L.(L.) amazonensis }\end{array}$} \\
\hline & E. coli & P. aeruginosa & S. aureus & B. cereus & \\
\hline 1 & 13,1 & $>26,2$ & $>26,2$ & 13,1 & $>0,3$ \\
\hline 2 & $>27,6$ & $>27,6$ & $>27,6$ & $>27,6$ & 0,137 \\
\hline 3 & $>33,1$ & $>33,1$ & $>33,1$ & $>33,1$ & $>0,3$ \\
\hline 4 & $>25$ & $>25$ & $>25$ & $>25$ & 0,099 \\
\hline 5 & 8,3 & 16,5 & 16,5 & 16,5 & $>0,3$ \\
\hline 6 & 11,2 & $>22,4$ & $>22,4$ & 11,2 & $>0,3$ \\
\hline 7 & $>25$ & $>25$ & $>25$ & $>25$ & $>0,3$ \\
\hline 8 & $>22,4$ & $>22,4$ & $>22,4$ & $>22,4$ & $>0,3$ \\
\hline 9 & $>26,2$ & $>26,2$ & $>26,2$ & $>26,2$ & $>0,3$ \\
\hline 10 & $>27,6$ & $>27,6$ & $>27,6$ & $>27,6$ & $>0,3$ \\
\hline Ampicilina & 0,018 & 0,072 & 0,009 & 0,036 & - \\
\hline Pentamidina & - & - & - & - & 0,015 \\
\hline Anfotericina B & - & - & - & - & 0,007 \\
\hline
\end{tabular}

(a) Determinação da concentração inibitória mínima (CIM) empregando-se o método de microdiluição de acordo com diretrizes da CLSI de M7-A6. Espécies utilizadas: Escherichia coli ATCC 1109219, Bacillus cereus MIP 96016, Pseudomonas aeruginosa ATCC 9027 e Staphylococcus aureus ATCC 6538; (b) Segundo metodologia descrita por Maia e colaboradores na referência 37, com adaptações.

resultados de $\mathrm{IC}_{50}$ inferiores a $10 \mu \mathrm{mol} \mathrm{L}^{-1}$ (cutoff definido pelo DNDi) são bons candidatos para estudos em leshimaniose visceral. Por outro lado, os próprios autores chamam atenção para o fato de que este critério não é aplicado rigorosamente. A escolha do cutoff nestes casos seria arbitrária, sempre correndo o risco de eliminar um composto com potencial atividade. Neste sentido, a extensão deste protocolo para a síntese de novas $\alpha$-acetiloxi- $N$-cicloexilamidas, derivadas dos compostos mais promissores para atividade leishmanicida, está em andamento em nosso laboratório, com vistas à obtenção de uma quimioteca maior de compostos potencialmente ativos para futuro estudo da relação estrutura-atividade.

\section{PARTE EXPERIMENTAL}

\section{Métodos gerais}

Todos e reagentes e solventes foram adquiridos de revendedores usuais (principalmente Sigma-Aldrich Co. e Labsynth) e utilizados sem purificação prévia. Atenção: cicloexilisonitrila tem odor muito forte e desagradável, além de ser tóxica, devendo ser manipulada com cuidado na capela de exaustão! As reações foram acompanhadas por cromatografia em camada delgada (CCD) utilizando cromatofolhas de sílica gel do tipo 60-F254/0,2 mm (Merck) e luz UV (254 nm), iodo, e/ou solução de ácido fosfomolíbdico para visualização. Os pontos de fusão (PF) foram medidos em equipamento Meting Point Buchi M-565 com termômetro não aferido. Os espectros de RMN de ${ }^{1} \mathrm{H}$ $\mathrm{e}^{13} \mathrm{C}$ foram obtidos utilizando os aparelhos Bruker AC-200, Bruker DPX-300, Bruker AVIII 400 e Varian INOVA 300. As amostras foram preparadas utilizando $\mathrm{CDCl}_{3}$ como solvente e TMS (tetrametilsilano) como padrão interno; os deslocamentos químicos $(\delta)$ são descritos em ppm. Os espectros de infravermelho (IV) foram obtidos em aparelho Shimadzu IR Prestige 21 (ATR); as frequências de absorção $\left(v_{\max }\right)$ foram expressas em $\mathrm{cm}^{-1}$. Os espectros de massas de baixa resolução (EMBR) foram obtidos em cromatógrafo gasoso acoplado a espectrômetro de massas (CG-EM) modelo PQ 2010 Plus da Shimadzu, com injetor AOC 20i também da Shimadzu. As análises elementares foram realizadas em equipamento Perkin-Elmer 2400/CHN. As reações sob aquecimento por micro-ondas foram realizadas em reator
Discover ${ }^{\circledR}$ da CEM, quando executadas em sistema aberto, ou em reator Microwave $300 \AA$ da Anton Paar quando em sistema fechado, utilizando modelos de frascos de reação (vials) disponibilizados pelos fabricantes.

\section{Procedimento geral para a preparação de $\alpha$-acetiloxi- $N$ - cicloexilamidas sob aquecimento convencional}

Em um balão equipado com condensador de refluxo, foram adicionados cicloexilisonitrila (1,0 mmol), ácido acético glacial (1,0 mmol) e o respectivo aldeído (1,0 mmol) em $\mathrm{CH}_{2} \mathrm{Cl}_{2}$ ou DMC $(5 \mathrm{~mL})$. A mistura reacional foi refluxada por $48 \mathrm{~h}$, quando então o solvente foi removido a pressão reduzida. O produto bruto foi purificado por coluna cromatográfica em sílica gel, com eluição de hexanos/AcOEt (1:1), fornecendo as $\alpha$-acetiloxi- $N$-cicloexilamidas 1-10 desejadas nos rendimentos descritos na Tabela 2 e Esquema 2.

\section{Procedimento geral para a preparação de $\alpha$-acetiloxi- $N$ - cicloexilamidas sob aquecimento por micro-ondas}

Em um vial de tamanho e formato apropriado (de acordo com modelo do fabricante do reator; CEM ou Anton Paar), foram adicionados cicloexilisonitrila (1,0 mmol), ácido acético glacial (1,0 mmol) e o orto-nitrobenzaldeído (1,0 mmol) em DMC $(5 \mathrm{~mL})$. A mistura reacional foi aquecida nas condições descritas na Tabela 3. Atenção: no caso da reação em frasco aberto a $90{ }^{\circ} \mathrm{C}$ (Tabela 3, entrada 4), foi conectado ao frasco reacional um condensador de refluxo. Após o tempo de reação especificado, o solvente foi removido a pressão reduzida e o produto bruto foi purificado por coluna cromatográfica em sílica gel, com eluição de hexanos/AcOEt (1:1), fornecendo a $\alpha$-acetiloxi- $N$-cicloexilamida 8 nos rendimentos descritos na Tabela 3 .

$\alpha$-(Acetiloxi)- $N$-cicloexil-benzenoacetamida (CAS \# 6273566-4) (1).$^{33,34}$ Sólido branco; P.F. $=122-124{ }^{\circ} \mathrm{C}$ (relatado: P.F. = $\left.125-126{ }^{\circ} \mathrm{C}\right) ; 3 \mathrm{IV}(\mathrm{KBr}) v_{\max } 3316,2929,1744,1681,1544,1238$ $\mathrm{cm}^{-1} ; \mathrm{RMN}^{1} \mathrm{H}\left(300 \mathrm{MHz}, \mathrm{CDCl}_{3}\right) \delta 7,45-7,35(\mathrm{~m}, 5 \mathrm{H}), 6,05(\mathrm{~s}, 1 \mathrm{H})$, $5,97(\mathrm{~d}, 1 \mathrm{H}, J=7,5 \mathrm{~Hz}), 3,83-3,78(\mathrm{~m}, 1 \mathrm{H}), 2,19$ (s, 3H), 1,93-1,88 (m, 2H), 1,78-1,58 (m, 3H), 1,47-1,11 (m, 5H); RMN ${ }^{13} \mathrm{C}(75 \mathrm{MHz}$, 
$\left.\mathrm{CDCl}_{3}\right) \delta 169,1,167,2,139,8,128,9,128,7,127,4,75,5,48,2,32,9$, 25,4, 24,7, 21,0; EMBR $m / z,(\%): 275,2(2,3)\left[\mathrm{M}^{+}\right], 276,6(2,7)$ $\left[(\mathrm{M}+\mathrm{H})^{+}\right], 108,1(100)$.

$\alpha$-(Acetiloxi)- $N$-cicloexil-4-metóxi-benzenoacetamida (CAS \# 841282-55-1) (2). ${ }^{35}$ Sólido branco. IV (KBr) $v_{\max } 3286,2933,1739$, $1656,1560,1224 \mathrm{~cm}^{-1} ; \mathrm{RMN}^{1} \mathrm{H}\left(400 \mathrm{MHz}, \mathrm{CDCl}_{3}\right) \delta 7,33-7,37(\mathrm{~m}$, $2 \mathrm{H}), 6,88-6,90(\mathrm{~m}, 2 \mathrm{H}), 6,01(\mathrm{~s}, 1 \mathrm{H}), 5,95(\mathrm{~d}, J=8.0 \mathrm{~Hz}, 1 \mathrm{H}), 3,80$ (s, 3H), 3,77-3,84 (m, 1H), 2,16 (s, 3H), 1,88-1,95 (m, 2H), 1,60-1,72 (m, 3H), 1,12-1,42 (m, 5H); RMN ${ }^{13} \mathrm{C}\left(100 \mathrm{MHz}, \mathrm{CDCl}_{3}\right) \delta 169,2$, $167,5,160,0,132,2,129,0,114,1,75,1,55,3,48,2,33,0,32,9,25,4$,

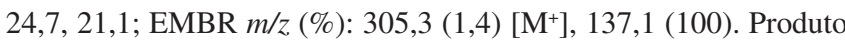
obtido em mistura (4:1) com o material de partida (determinado por $\left.\mathrm{RMN}{ }^{1} \mathrm{H}\right)$

$\alpha$-(Acetiloxi)- $N$-cicloexil-4-nitro-benzenoacetamida (3). Sólido levemente amarelado; IV (KBr) $v_{\max } 3280,2927,1753,1656,1560$, $1521,1371,1224 \mathrm{~cm}^{-1} ; \mathrm{RMN}^{1} \mathrm{H}\left(300 \mathrm{MHz}, \mathrm{CDCl}_{3}\right) \delta 8,20-8,25(\mathrm{~m}$, 2H), 7,60-7,65 (m, 2H), 6,14, (sl, H-NH), 6,11, s, 1H), 3,74-3,81 (m, 1H), 2,24 (s, 3H), 1,88-1,94 (m, 2H), 1,60-1,73 (m, 3H), 1,11-1,44 (m, $5 \mathrm{H}) ; \mathrm{RMN}^{13} \mathrm{C}\left(75 \mathrm{MHz}, \mathrm{CDCl}_{3}\right) \delta 168,8,165,9,148,1,142,7,128,0$, 123,8, 74,3, 48,5, 32,9, 25,4, 24,7, 20,9; EMBR m/z (\%): 321,1 (0,3) $\left[(\mathrm{M}+\mathrm{H})^{+}\right], 153,1(100) ; \mathrm{AE}$ calculado para $\mathrm{C}_{16} \mathrm{H}_{20} \mathrm{~N}_{2} \mathrm{O}_{5}: \mathrm{C}(59,99), \mathrm{H}$ $(6,29), \mathrm{N}(8,74)$; encontrado: C $(60,27), \mathrm{H}(6,18), \mathrm{N}(8,52)$.

$\alpha$-(Acetiloxi)- $N$-cicloexil-4-bromo-benzenoacetamida (4). Sólido branco; P.F. $=158-160{ }^{\circ} \mathrm{C}$; IV (KBr) $v_{\max } 3286,2929,1751$, $1656,1554,1232 \mathrm{~cm}^{-1} ; \mathrm{RMN}{ }^{1} \mathrm{H}\left(200 \mathrm{MHz}, \mathrm{CDCl}_{3}\right) \delta 7,39-7,45(\mathrm{~m}$, $2 \mathrm{H}), 7,20-7,26(\mathrm{~m}, 2 \mathrm{H}), 6,00(\mathrm{~d}, J=8,2 \mathrm{~Hz}, 1 \mathrm{H}), 5,91(\mathrm{~s}, 1 \mathrm{H}), 3,61-$ $3,79(\mathrm{~m}, 1 \mathrm{H}), 2,11$ (s, 3H), 1,52-1,85 (m, 5H), 0,97-1,39 (m, 5H); $\mathrm{RMN}{ }^{13} \mathrm{C}\left(50 \mathrm{MHz}, \mathrm{CDCl}_{3}\right) \delta 168,9,166,7,134,8,131,8,129,0,123,0$, $74,7,48,2,32,9,32,8,25,4,24,7,21,0 ; \operatorname{EMBR} m / z(\%): 274,1(5,9)$ $\left[(\mathrm{M}-\mathrm{Br})^{+}\right], 43,1$ (100); AE calculado para $\mathrm{C}_{16} \mathrm{H}_{20} \mathrm{BrNO}_{3}: \mathrm{C}(54,25)$, $\mathrm{H}(5,69), \mathrm{N}(3,95), \mathrm{Br}(22,56)$; encontrado: C $(54,27), \mathrm{H}(5,66), \mathrm{N}$ $(3,88), \mathrm{Br}(22,58)$.

$\alpha$-(Acetiloxi)- $N$-cicloexil-pentanamida (5). Sólido branco; P.F. $=80-83{ }^{\circ} \mathrm{C} ; \mathrm{IV}(\mathrm{KBr}) v_{\max } 3277,2933,1741,1651,1554,1234 \mathrm{~cm}^{-1}$; $\mathrm{RMN}{ }^{1} \mathrm{H}\left(200 \mathrm{MHz}, \mathrm{CDCl}_{3}\right) \delta 5,90(\mathrm{~d}, J=7,6 \mathrm{~Hz}, \mathrm{H}-\mathrm{NH}), 5,10(\mathrm{t}, J$ $=6,0 \mathrm{~Hz}, 1 \mathrm{H0} ; 3,71-3,83(\mathrm{~m}, 1 \mathrm{H}), 2,13(\mathrm{~s}, 3 \mathrm{H}), 1,57-1,91(\mathrm{~m}, 7 \mathrm{H})$, 1,04-1,46 (m, 7H), 0,86 (t, $J=7,2 \mathrm{~Hz}, 3 \mathrm{H})$; RMN ${ }^{13} \mathrm{C}(50 \mathrm{MHz}$, $\left.\mathrm{CDCl}_{3}\right) \delta 169,6,168,8,73,9,33,9,33,0,32,9,25,4,24,7,20,9,17,9$, 13,7; EMBR $m / z(\%): 241,1(1,3)\left[\mathrm{M}^{+}\right], 242,1(2,1)\left[(\mathrm{M}+\mathrm{H})^{+}\right], 43,0$ (100); AE calculado para $\mathrm{C}_{13} \mathrm{H}_{23} \mathrm{NO}_{3}$ : C $(64,70), \mathrm{H}(9,61), \mathrm{N}(5,80)$; encontrado: C $(65,09), \mathrm{H}(9,21), \mathrm{N}(5,83)$.

$\alpha$-(Acetiloxi)- $N$-cicloexil-3-metil-butanamida (CAS \# 92158 54-8) (6). ${ }^{36}$ Sólido branco; P.F. $=116-118{ }^{\circ} \mathrm{C}$ (relatado: P.F. $=121-$ $\left.122{ }^{\circ} \mathrm{C}\right) ;^{36} \mathrm{IV}(\mathrm{KBr}) v_{\max } 3290,2935,1737,1653,1558,1236 \mathrm{~cm}^{-1}$; $\mathrm{RMN}{ }^{1} \mathrm{H}\left(400 \mathrm{MHz}, \mathrm{CDCl}_{3}\right) \delta 5,79(\mathrm{~d}, J=6,5 \mathrm{~Hz}, 1 \mathrm{H}), 5,00(\mathrm{~d}, J$ $=5,3 \mathrm{~Hz}, 1 \mathrm{H}), 3,76-3,84(\mathrm{~m}, 1 \mathrm{H}), 2,23-2,34(\mathrm{~m}, 1 \mathrm{H}), 2,16(\mathrm{~s}, 3 \mathrm{H})$, 1,86-1,93 (m, 2H), 1,60-1,71 (m, 3H), 1,33-1,42 (m, 2H), 1,09-1,21 $(\mathrm{m}, 3 \mathrm{H}), 0,92-0,95(\mathrm{~m} 6 \mathrm{H})$; RMN ${ }^{13} \mathrm{C}\left(100 \mathrm{MHz}, \mathrm{CDCl}_{3}\right) \delta 169,7$, 168,2, 78,1, 47,8, 33,1, 32,9, 30,5, 25,4, 24,7, 20,9, 18,7, 16,9; EMBR $m / z(\%): 241,2(3,4)\left[\mathrm{M}^{+}\right], 43,1(100)$.

$\alpha$-(Acetiloxi)- $N$-cicloexil-2-metóxi-benzenoacetamida (7). Sólido levemente amarelado; P.F. $=112-113^{\circ} \mathrm{C}$; IV (KBr) $v_{\max } 3265$, 2933, 1739, 1664, 1552, $1242 \mathrm{~cm}^{-1} ; \mathrm{RMN}{ }^{1} \mathrm{H}\left(500 \mathrm{MHz}, \mathrm{CDCl}_{3}\right) \delta$ 7,48 (dd, $J=1,6$ e 7,6 Hz, 1H), 7,32 (ddd, $J=1,6,7,6$ e $8,2 \mathrm{~Hz}, 1 \mathrm{H}$ ), $6,99(\mathrm{td}, J=1,0$ e $7,6 \mathrm{~Hz}, 1 \mathrm{H}), 6,92(\mathrm{~d}, J=8,5 \mathrm{~Hz}, 1 \mathrm{H}), 6,34$ (s, $1 \mathrm{H}), 6,04$ (d, $J=8,5 \mathrm{~Hz}, 1 \mathrm{H}), 3,89$ (s, 3H), 3,72-3,78 (m, 1H), 2,190 (s, 3H), 1,93-1,96 (m, 1H), 1,56-1,78 (m, 4H), 1,03-1,41 (m, 5H);
$\mathrm{RMN}{ }^{13} \mathrm{C}\left(125 \mathrm{MHz}, \mathrm{CDCl}_{3}\right) \delta 169,9,167,5,156,3,129,9,128,5$, 124,2, 121,1, 110,9, 70,2, 55,6, 47,9, 32,9, 32,6, 25,5, 24,5, 24,4, 21,0; EMBR $m / z$ (\%): 305,1 $(1,2)\left[\mathrm{M}^{+}\right], 137,1$ (100); AE calculado $\mathrm{C}_{17} \mathrm{H}_{23} \mathrm{NO}_{4}$ : C $(66,86), \mathrm{H}(7,59), \mathrm{N}(4,59)$; encontrado: $\mathrm{C}(67,11), \mathrm{H}$ $(7,49), \mathrm{N}(4,38)$.

$\alpha$-(Acetiloxi)- $N$-cicloexil-2-nitro-benzenoacetamida (8). Sólido levemente amarelado; P.F. $=129-131{ }^{\circ} \mathrm{C}$; IV $(\mathrm{KBr}) v_{\max } 3242,2933$, $1749,1653,1529,1363,1224 \mathrm{~cm}^{-1}$; RMN ${ }^{1} \mathrm{H}\left(300 \mathrm{MHz}, \mathrm{CDCl}_{3}\right) \delta$ $7,98$ (dd, $J=1,3$ e $8,1 \mathrm{~Hz}, 1 \mathrm{H}), 7,79$ (dd, $J=1,4$ e 7,8 Hz, 1H), 7,66 (td, $J=1,3$ e 7,6 Hz, 1H), 7,51 (ddd, $J=1,3,7,4$ e $8,1 \mathrm{~Hz}, 1 \mathrm{H}), 6,58$ (s, 1H), 6,28 (d, $J=7,3 \mathrm{~Hz}, 1 \mathrm{H}), 3,71-3,81(\mathrm{~m}, 1 \mathrm{H}), 2,18(\mathrm{~s}, 3 \mathrm{H})$, $1,56-1,97(\mathrm{~m}, 5 \mathrm{H}), 1,08-1,38(\mathrm{~m}, 5 \mathrm{H})$; RMN ${ }^{13} \mathrm{C}\left(75 \mathrm{MHz}, \mathrm{CDCl}_{3}\right)$ $\delta 169,5,165,7,148,1,133,6,130,8,129,9,129,5,124,7,71,0,48,5$, 32,6, 25,4, 24,6, 20,7; EMBR m/z (\%): 274,0 (5,9) [(M-NO $\left.)^{+}\right], 149,1$ (100); AE calculado $\mathrm{C}_{16} \mathrm{H}_{20} \mathrm{~N}_{2} \mathrm{O}_{5}$ : C $(59,99), \mathrm{H}(6,29), \mathrm{N}(8,74)$; encontrado: C $(60,02), \mathrm{H}(6,25), \mathrm{N}(8,67)$.

$\alpha$-(Acetiloxi)- $N$-cicloexil-2-bromo-benzenoacetamida (9). Sólido levemente amarelado; P.F. $=129-133^{\circ} \mathrm{C}$; IV (KBr) $v_{\max } 3273$, $2927,1745,1656,1558,1228,1037 \mathrm{~cm}^{-1} ; \mathrm{RMN}^{1} \mathrm{H}\left(500 \mathrm{MHz}, \mathrm{CDCl}_{3}\right)$ $\delta 7,58(\mathrm{~d}, J=7,9 \mathrm{~Hz}, 1 \mathrm{H}), 7,53(\mathrm{dd}, J=1,3$ e 7,6 Hz, 1H), 7,35 (t, $J$ $=7,6 \mathrm{~Hz}, 1 \mathrm{H}), 7,22(\mathrm{td}, J=1,6 \mathrm{e} 7,7 \mathrm{~Hz}, 1 \mathrm{H}), 6,31(\mathrm{~s}, 1 \mathrm{H}), 5,96(\mathrm{~d}$, $J=7,3 \mathrm{~Hz}, 1 \mathrm{H}), 3,76-3,81(\mathrm{~m}, 1 \mathrm{H}), 1,93-1,97(\mathrm{~m}, 1 \mathrm{H}), 1,80-1,85$ (m, 1H), 1,58-1,71 (m, 3H), 1,08-1,40 (m, 5H); RMN ${ }^{13} \mathrm{C}(125 \mathrm{MHz}$, $\left.\mathrm{CDCl}_{3}\right) \delta 169,6,166,3,135,1,133,0,130,3,129,5,127,9,123,3$, 74,4, 48,3, 32,7, 32,6, 25,4, 24,5, 24,6, 20,8; EMBR m/z (\%): 274,3 $(18,5)\left[(\mathrm{M}-\mathrm{Br})^{+}\right], 149,1(100) ; \mathrm{AE}$ calculado $\mathrm{C}_{16} \mathrm{H}_{20} \mathrm{BrNO}_{3}: \mathrm{C}(54,25)$, $\mathrm{H}(5,69), \mathrm{N}(3,95), \mathrm{Br}(22,56)$; encontrado: C $(54,24), \mathrm{H}(5,69), \mathrm{N}$ $(3,89), \operatorname{Br}(23,05)$.

$\alpha$-(Acetiloxi)- $\boldsymbol{N}$-cicloexil-2-metil-benzenoacetamida (10). Sólido levemente amarelado; P.F. $=110-113^{\circ} \mathrm{C}$; IV $(\mathrm{KBr}) v_{\max } 3265$, 2931, 1739, 1653, 1564, $1232 \mathrm{~cm}^{-1} ; \mathrm{RMN}^{1} \mathrm{H}\left(300 \mathrm{MHz}, \mathrm{CDCl}_{3}\right) \delta$ 7,19-7,33 (m, 54H), 6,29 (s, 1H), 5,92 (d, $J=7,7 \mathrm{~Hz}, 1 \mathrm{H}), 3,76-3,86$ (m, 1H), 2,46, (s, 3H), 2,17 (s, 3H0, 1,89-1,95 (m, 2H), 1,65-1,72 (m, 3H), 1,10-1,45 (m, 5H); RMN ${ }^{13} \mathrm{C}\left(75 \mathrm{MHz}, \mathrm{CDCl}_{3}\right) \delta 169,3$, $167,5,137,2,134,3,130,9,128,9,127,5,126,2,73,0,48,2,32,9,25,4$,

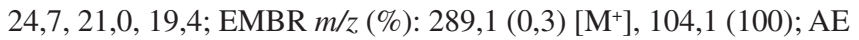
calculado $\mathrm{C}_{17} \mathrm{H}_{23} \mathrm{NO}_{3}$ : $\mathrm{C}(70,56), \mathrm{H}(8,01), \mathrm{N}(4,84)$; encontrado: $\mathrm{C}$ $(70,58), \mathrm{H}(7,81), \mathrm{N}(4,64)$. Traço do material de partida pode ser verificado nos espectros de $\mathrm{RMN}{ }^{1} \mathrm{H} \mathrm{e}{ }^{13} \mathrm{C}$ do produto; amostra analítica foi obtida após uma segunda coluna cromatográfica (sílica gel 70-230 Mesh) com eluição de hexano/AcOEt (1/1).

\section{Teste de atividade antimicrobiana}

A atividade antibacteriana das $\alpha$-acetiloxi- $N$-cicloexilamidas 1-10 foi investigada empregando-se o método de microdiluição, de acordo com as diretrizes da CLSI de M7-A6. O ensaio foi realizado com quatro espécies bacterianas diferentes: Escherichia coli ATCC 1109219 (American Type Culture Collection), Bacillus cereus MIP 96016, Pseudomonas aeruginosa ATCC 9027 e Staphylococcus aureus ATCC 6538. Agar e caldo Mueller-Hinton (Difco Laboratories, Detroit, EUA) foram utilizados para o crescimento bacteriano. O inóculo foi feito a partir de culturas de cada um dos micro-organismos crescidos em caldo Mueller-Hinton, durante a noite, à temperatura de $37{ }^{\circ} \mathrm{C}$. O inóculo foi diluído no mesmo meio até uma concentração final de aproximadamente $10^{8} \mathrm{UFC} / \mathrm{mL}$. Os compostos foram dissolvidos em DMC e diluídos com caldo Mueller-Hinton até uma concentração de 30-25 mmol L-1. Outras diluições em série 1:2 foram realizadas por adição de caldo Mueller-Hinton para atingir uma concentração final de 30 a 0,234 mmol L-1. Em seguida, um volume 
de $100 \mu \mathrm{L}$ de cada diluição foi distribuído em placas de 96 poços e em cada poço foi inoculado $5 \mu \mathrm{L}$ de uma suspensão bacteriana $\left(10^{8}\right.$ $\mathrm{UFC} / \mathrm{mL}$ ). Para o controle do crescimento e da esterilidade utilizou-se apenas meio sem adição do composto e solvente. Como controle da toxicidade do solvente foi utilizado cultura com DMC. Utilizou-se ampicilina (Sigma-Aldrich; 0,288-0,0023 $\mathrm{mmol} \mathrm{L}^{-1}$ ) como controle antibacteriano positivo. Todos os experimentos foram realizados em triplicata e as placas de microdiluição foram incubadas a $37{ }^{\circ} \mathrm{C}$ durante $18 \mathrm{~h}$. Os valores de CIM (Concentração Mínima Inibitória) foram definidos como a menor concentração de cada composto capaz de inibir completamente o crescimento dos micro-organismos testados. Os resultados para MIC de 1-10 estão expressos em mmol $\mathrm{L}^{-1}$ e descritos na Tabela 4.

\section{Estudos de atividade leishmanicida}

A avaliação da atividade leishmanicida in vitro foi realizada segundo metodologia descrita por Maia e colaboradores, ${ }^{37}$ com algumas modificações. Formas promastigotas de L. amazonensis foram cultivadas em meio 199 (Sigma-Aldrich) suplementado com 10,0\% (v/v) de soro fetal bovino (Gibco) e 1,0\% de penicilina (10.000 UI/mL)/ estreptomicina $(10,0 \mathrm{mg} / \mathrm{mL}$ ) (Sigma-Aldrich). Os parasitas coletados na fase $l o g$ de crescimento foram contados em câmara de Neubauer e a concentração do inóculo ajustada para $2 \times 10^{6}$ parasitas $/ \mathrm{mL}$. Então, $40 \mu \mathrm{L}$ da solução contendo os parasitas foram plaqueados em placas de 96 poços na presença ou ausência de diferentes concentrações (diluição seriada) das $\alpha$-acetiloxi- $N$-cicloexilamidas 1-10. A diluição seriada dos compostos foi realizada em 6 passos de diluição sendo que cada passo foi preparado em duplicata, cobrindo uma variação de concentração de 0,3 a $0,00937 \mathrm{mmol} \mathrm{L}^{-1}$ para cada composto. Após $48 \mathrm{~h}$ de incubação a $26^{\circ} \mathrm{C}$, as placas foram inspecionadas em microscópio óptico invertido tanto para verificar o crescimento dos parasitas como para avaliar ausência de contaminação. Em seguida, a viabilidade celular foi verificada adicionando $10 \mu \mathrm{L}$ de solução de resazurin (PrestoBlueTM, Life Technologies, Carlsbad, CA, USA) em cada poço e as placas incubadas por $2 \mathrm{~h}$ a $26{ }^{\circ} \mathrm{C}$. A leitura das placas foi realizada em espectrofluorímetro (Biotek, Instruments, Inc., Winooski, VT, USA) com excitação de 560 nm e emissão de 590 $\mathrm{nm}$. Os dados foram analisados usando o software GraphPad Prism (La Jolla, CA, USA). A diminuição da fluorescência (inibição) foi expressa como porcentagem da fluorescência das culturas controles (sem adição de compostos) e este valor considerado a porcentagem

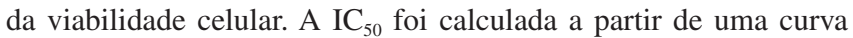
de inibição sigmoidal na qual o eixo $\mathrm{Y}$ corresponde a porcentagem da viabilidade celular e o eixo $\mathrm{X}$ o $\log$ da concentração dos novos análogos. Anfotericina B e pentamidina foram adicionadas a cada placa para utilização como drogas de referência. Os resultados de $\mathrm{IC}_{50}$ para 1-10 estão expressos em mmol L $\mathrm{L}^{-1}$ e apresentados na Tabela 4.

\section{MATERIAL SUPLEMENTAR}

Espectros de Ressonância Magnética Nuclear de ${ }^{1} \mathrm{H}$ e de ${ }^{13} \mathrm{C}$ das $\alpha$-acetiloxi- $N$-cicloexilamidas 1-10 deste estudo encontram-se disponíveis em http://quimicanova.sbq.org.br, na forma de arquivo PDF, com acesso livre.

\section{AGRADECIMENTOS}

À Fundação de Amparo à Pesquisa do Estado de São Paulo (FAPESP; projetos no 2007/07338-6 e 2011/12821-3) e ao Conselho Nacional de Desenvolvimento Científico e Tecnológico (CNPq) pelas bolsas concedidas a B. R. Oliveira e C. C. Silva.

\section{REFERÊNCIAS}

1. Multicomponent Reactions; Zhu, J.; Bienaymé, H.; eds. Wiley-VCH: Weinheim, 2005.

2. Strecker, A.; Ann. Chem. Pharm. 1850, 75, 27; Strecker, A.; Ann. Chem. Pharm. 1854, 91, 349.

3. Biginelli, P.; Ber. Dtsch. Chem. Ges. 1891, 24, 1317; Biginelli, P.; Ber. Dtsch. Chem. Ges. 1891, 24, 2962.

4. Hantzsch, A.; Justus Liebigs Ann. Chem. 1882, $215,1$.

5. Mannich, C.; Krösche, W.; Arch. Pharm. 1912, $250,647$.

6. Eckert, H.; Molecules 2012, 17, 1074; Ruijter, E.; Scheffelaar, R.; Orru, R. V. A.; Angew. Chem., Int. Ed. 2011, 51, 6234; Ismbert, N.; Lavilla, R.; Chem. Eur. J. 2008, 14, 8444.

7. Mironov, M.A.; Russ. J. Gen. Chem. 2010, 80, 2628.

8. Shaaban, S.; Abdel-Wahab, B. F.; Mol. Divers. 2016, 20, 233; Devi, N.; Rawal, R. K.; Singh, V.; Tetrahedron 2015, 71, 183; Khan, M. M.; Yousuf, R.; Shafiullah, S. K.; RSC Adv. 2015, 5, 57883; Liu, Z.-Q.; Curr. Org. Synth. 2015, 12, 20; Garbarino, S.; Protti, S.; Basso, A. Synthesis 2015, 47, 2385; Alvim, H. G. O.; Silva Jr., E. N.; Neto, B. A. D.; RSC Adv. 2014, 4, 54282; Brauch, S.; van Berkel, S. S.; Westermann, B.; Chem. Soc. Rev. 2013, 42, 4948; Dömling, A.; Wang, W.; Wang, K.; Chem. Rev. 2012, 112, 3083; Isambert, N.; Duque, M. M. S.; Plaquevent, J.-C.; Génisson, Y.; Rodriguez, J.; Constantieux, T.; Chem. Soc. Rev. 2011, 40, 1347.

9. Cioc, R. C.; Ruijter, E.; Orru, R. V. A.; Green Chem. 2014, 16, 2958.

10. Gu, Y.; Green Chem. 2012, 14, 2091.

11. Váradi, A.; Palmer, T. C.; Dardashti, R. N.; Majumdar, S.; Molecules 2016, 21, 19; Eckert, H.; Molecules 2012, 17, 1074; Sadjadi, S.; Heravi, M. M.; Tetrahedron 2011, 67, 2707; Lygin, A. V.; de Meijere A.; Angew. Chem., Int. Ed. 2010, 49, 9094; Sunderhaus, J. D.; Martin, S. F.; Chem. Eur. J. 2009, 15, 1300.

12. El Kaïm, L.; Grimaud, L.; Eur. J. Org. Chem. 2014, 7749; Koopmanschap, G.; Rujiter, E.; Orru, R. V. A.; Beilstein J. Org. Chem. 2014, 10, 544; Qiu, G.; Ding, Q.; Wu, J.; Chem. Soc. Rev. 2013, 42, 5257; Heravi, M. M.; Maghimi, S.; J. Iran. Chem. Soc. 2011, 8, 306; Dömling, A.; Chem Rev. 2006, 106, 17.

13. Passerini, M.; Gazz. Chim. Ital. 1921, 51, 126.

14. Ugi, I.; Steinbruckner, C.; Angew. Chem. 1960, 72, 267.

15. Kazemizadeh, A. R.; Ramazani, A.; Curr. Org. Chem. 2012, 16, 418.

16. Gola, G. F.; Di Venosa, G. M.; Sáenz, D. A.; Calvo, G. H.; Cabrera, G. M.; Casas, A. G.; Ramírez, J. A.; RSC Adv. 2016, 6, 89492; Wessjohann, L. A.; Morejón, M. C.; Ojeda, G. M.; Rhoden, C. R. B.; Rivera, D. G.; J. Org. Chem. 2016, 81, 6535; Li, R.-K.; Yang, Q.-L.; Liu, Y.; Li, D.-W.; Huang, N.-Y.; Liu, M.-G.; Chin. Chem. Lett. 2016, 27, 345; PolindaraGarcía, L.; Juaristi, E.; Eur. J. Org. Chem. 2016, 1095; Chandgude, A. L.; Dömling, A. Green Chem. 2016, 18, 3718; Chandgude, A. L.; Dömling, A. Org. Lett. 2016, 18, 6396.

17. Constable, D. J. C.; Jimenez-Gonzalez, C.; Henderson, R. K.; Org. Process Res. Dev. 2007, 11, 133.

18. Alfonsi, K.; Colberg, J.; Dunn, P. J.; Fevig, T.; Jennings, S.; Johnson, T. A.; Kleine, H. P.; Knight, C.; Nagy, M. A.; Perry, D. A.; Stefaniak, M.; Green Chem. 2008, 10, 31 .

19. Henderson, R. K.; Jiménez-González, C.; Constable, D. J. C.; Alston, S. R.; Inglis, G. G. A.; Fisher, G.; Sherwood, J.; Binks, S. P.; Curzons, A. D.; Green Chem. 2011, 13, 854.

20. Prat, D.; Pardigon, O.; Flemming, H.-W.; Letestu, S.; Ducandas, V.; Isnard, P.; Guntrum, E.; Senac, T.; Ruisseau, S.; Cruciani, P.; Hosek, P.; Org. Process Res. Dev. 2013, 17, 1517.

21. Schäffner, B.; Schäffner, F.; Verevkin, S. P.; Börner, A.; Chem. Rev. 2010, 110, 4554.

22. Aricò, F.; Tundo, P.; Russ. Chem. Rev. 2010, 79, 479.

23. Dimetilcarbonato: CAS\#616-38-6; Dietilcarbonato: CAS\#105-58-8; Etileno carbonato: CAS\#96-49-1; Propileno carbonato: CAS\#108-32-7. 
24. MacMillan, D. S.; Murray, J.; Sneddon, H. F.; Jamiesona, C.; Watson, A. J. B.; Green Chem. 2013, 15, 596; Keraania, A.; Fischmeisterb, C.; Renouarda, T.; Le Flochc, M.; Baudrya, A.; Bruneaub, C.; RabillerBaudrya, M.; J. Mol. Catal. A: Chem. 2012, 357, 73; Miao, X.; Fischmeister, C.; Bruneau, C.; Dixneuf, P. H.; ChemSusChem 2008, 1, 813.

25. Barreto, A. F. S.; Vercillo, O. E.; Andrade, C. K. Z.; J. Braz. Chem. Soc. 2011, 22, 462 .

26. Ingold, M.; López, G. V.; Porcal, W.; ACS Sustainable Chem. Eng. 2014, 2, 1093.

27. Souza, R. O. M. A.; Miranda, L. S. M.; Quim. Nova 2011, 34, 497; Strauus, C. R. Aust. J. Chem. 2009, 62, 3; Kappe, C. O.; Chem. Soc. Rev. 2008, 37, 1127; de la Hoz, A.; Díaz-Ortiz, A.; Moreno, A.; Chem. Soc. Rev. 2005, 34, 164; Kappe, C. O.; Angew. Chem., Int. Ed. 2004, 43, 6250.

28. Dömling, A.; Ugi, I.; Angew. Chem., Int. Ed. 2009, 39, 3168; Ramozzi, R.; Morokuma, K.; J. Org. Chem. 2015, 80, 5652.

29. Ugi, I.; Angew. Chem., Int. Ed. 1962, 1, 8.
30. Pirrung, M.C.; Das Sarma, K.; Wang, J.; J. Org. Chem. 2008, 73, 8723; Okandeji, B. O.; Sello, J. K.; J. Org. Chem. 2009, 74, 5067; Selaa, T.; Vigaloka, A.; Adv. Synth. Catal. 2012, 354, 2407; Li, M.; Qiu, B.; Kong, X.-J.; Wen, L.-R.; Org. Chem. Front. 2015, 2, 1326; Li, R.-K.; Yang, Q.-L.; Liu, Y.; Li, D.-W.; Huang, N.-Y.; Liu, M.-G.; Chin. Chem. Lett. 2016, 27, 345

31. Maeda, S.; Komagawa, S.; Uchiyama, M.; Morokuma, K.; Angew. Chem., Int. Ed. 2011, 50, 644.

32. Don, R.; Ioset J. R.; Parasitology 2014, 141, 140.

33. Iriuchijima, S.; Maniwa, K.; Tsuchihashi, G.-I.; Agr. Biol. Chem. 1976, 40, 2389.

34. Piersanti, G.; Remi, F.; Fusi, V.; Formica, M.; Giorgi, L.; Zappia, G.; Org. Lett. 2009, 11, 417.

35. El Mamouni, El H.; dos Santos, A.; El Kaim, L.; Ilitki, H.; Synlett 2016, 27, 2571.

36. McFarland, J. W.; J. Org. Chem. 1963, 28, 2179.

37. Maia, C.; Nunes, M.; Marques, M.; Henriques, S.; Rolão, N.; Campino, L.; Exp. Parasitol. 2013, 135, 36. 